\section{Entwicklung und Praxis- relevanz von Leitlinien in der stationären Depressionsbehandlung bei Kindern und Jugendlichen}

O. Bilke, S. Kühnel
Der von den Leitlinien abweichende Praktiker oder Kliniker sollte seine divergierende Meinung begründen können, ggf. sogar unter Zuziehung anderer Leitlinien mit höherer evidenzbasierter Relevanz (gemäss den Prinzipien der Evidence-based Medicine, z.B. [2]) im jeweiligen Einzellfall.

Die Entwicklung von Leitlinien ist nach allgemeinem Konsens eine vornehme Aufgabe unabhängiger wissenschaftlicher Fachgesellschaften, da besonders beim Einsatz von Medizintechnologie und Pharmaka Partikularinteressen von Sponsoren oder Anbietern nicht die Empfehlungen verfälschen dürfen.

\section{Leitlinienentwicklung in der Kinder- und Jugend- psychiatrie im deutschsprachigen Raum}

Durch die Routinebenutzung des multiaxialen Klassifikationsschemas für psychische Störungen des Kindes- und Jugendalters [3] im Rahmen der ICD-10 (WHO) ist die Kinder- und Jugendpsychiatrie seit vielen Jahren mit einer standardisierten Diagnostik auf sechs Betrachtungsebenen vertraut.

Stärker verhaltensorientierte Therapieansätze beiLeitlinien haben in den biopsychosozialen Gebieten der Medizin insbesondere in der Diagnostik und Epidemiologie eine lange Tradition. In der Kinderund Jugendpsychiatrie gilt seit über einem Jahrzehnt das Multiaxiale Klassifikationsschema (MAS/ICD-10) als international verbindlich. Seit etwa vier Jahren stehen von wissenschaftlichen Institutionen, Kliniken und Praktikern gemeinsam erarbeitete Leitlinien auch zu differentialdiagnostischen und therapeutischen Fragen für Deutschland zur Verfügung. Anhand der Leitlinie für die schwere episodische Depression wird die Entwicklung und die Praxisrelevanz kritisch diskutiert und eine Mitgestaltung der Kliniker an solchen Entwicklungen im Rahmen des Qualitätsmanagements beispielhaft dargestellt.

\section{Die Idee der Leitlinie im Qualitätsmanagement}

Leitlinien gehören ebenso wie beispielsweise Standards oder Outcome-Messungen zu den selbstverständlichen Bestandteilen verschiedener Qualitätssicherungssysteme. Sowohl im EFQM-Modell [1] als auch bei den ISO-Normen sind Leitlinien insbesondere im Sektor Prozessqualität vorgesehen. Leitlinien unterscheiden sich dabei klar von juristisch relevanten Richtlinien oder gar Gesetzesvorgaben. Sie stellen meist einen auf einem mehr oder weniger standardisierten und anspruchsvollen Expertenkonsens beruhenden literaturgestützten Empfehlungskatalog dar.

Korrespondenz:

Dr. med. Oliver Bilke

Klinik für Psychiatrie und Psychotherapie Littenheid

Bereich Jugendpsychiatrie

CH-9573 Littenheid

E-mail: o.bilke@klinik-littenheid.ch störung (ADS, auch ADD/H, ICD-10-Nr. F90.0) führten auch auf der Seite der Behandlung in Deutschland zu einem Bedürfnis nach Manualisierung und Entwicklung fachbezogener Standards und Leitlinien zu den bedeutenden Störungsbildern des Fachgebiets (schon früh hierzu: [4]). Die Anregungen der Arbeitsgemeinschaft der medizinisch-wissenschaftlichen Fachgesellschaften (AMWF) und die sukzessive Publikation der Guidelines der American Association of Child and Adolescent Psychiatry waren zusätzlicher äusserer Anlass zu einem umfangreichen Prozess der Leitlinienentwicklung, an dem insgesamt über 100 Fachpersonen aus Universitäten, Versorgungskliniken und Praxen beteiligt waren.

Diese Entwicklung begann offiziell bei einer Initialveranstaltung in Frankfurt 1996 [5] unter dem Leitmotiv "Von der Qualitätssicherung zur Lebensqualität». Nach der Online-Publikation 1999 (amwf. leitlinien@med.uni-duesseldorf.de) war der vorläufige Endpunkt die Buchveröffentlichung aller 34 Leitlinien im Jahre 2000 im Dt. Ärzteverlag [6]. Zurzeit erfolgt im Rahmen der Europäischen Arbeitsgemeinschaft für Dokumentation und Qualitätssicherung eine Überarbeitung und gegebenenfalls eine zweite aktualisierte Auflage.

Aufgrund regionaler Unterschiede und geringerer personeller Ressourcen, aber auch anderer therapeutischer Traditionen und Grundhaltungen ist eine analoge Entwicklung in der schweizerischen, aber auch der österreichischen Kinder- und Jugendpsychiatrie erst in den Anfängen, so dass die Leitlinien der drei deutschen Fachgesellschaften als die zur Zeit einzigen deutschsprachigen Qualitätssicherungselemente dieser Art gelten können.

Es stellt sich trotz aller unbestreitbaren Verdienste dieser Entwicklung aber für den Anwender die Frage nach klinischer und praktischer Relevanz und auch spielsweise bei der Aufmerksamkeitskonzentrations- 
zur Praktikabilität im Alltag, dies besonders in Zeiten allenthalben zunehmender Reglementierung und externer Kontrolle.

\section{Die Leitlinie F32/F33 (episodische Depression) als klinisches Beispiel}

Da der Erstautor dieses Beitrages in den Jahren 1996-1999 an der Entwicklung der Leitlinie F32/F33 unter der verantwortlichen Koordination von Prof. Dr. med. Ulrich Knölker an der medizinischen Universität zu Lübeck beteiligt war, lag es nahe, aus der jetzigen Sicht des klinisch und stationär tätigen Jugendpsychiaters die Bedeutung in der Alltagsarbeit anhand dieser Leitlinie zu analysieren.

Während leicht- und mittelgradige Depressionsformen wie z.B. die Dysthymia (F34.1) häufige und unterdiagnostizierte Störungen sind, stellt die episodische Depression eine seltene Diagnose bei schwerst, teilweise auch psychotisch (F32.3/F33.3) gestörten Jugendlichen (seltenst Kindern) dar. Deren Behandlung bleibt oft stationären Zentren vorbehalten. Bei diesen Fällen besteht zumeist eine erhebliche Komorbidität mit Angst- und Zwangsstörungen, mit Persönlichkeitsstörungen, Drogenabusus, Essstörungen, posttraumatischen Störungen und auch abnormen psychosozialen Umständen im Sinne der Achse fünf der MAS.

In der klinischen Arbeit ist daher diese Leitlinie vor allem im Bereich der Differentialdiagnose und der Differentialindikation ein wertvolles Hilfsmittel, das allerdings wegen der vorher beschriebenen Komorbidität besonders im Bereich der (Pharmako-)Therapie nur Hinweise geben kann. Beispielhaft kann hier die Diskussion um die Bedeutung des Johanniskrauts auch in der Behandlung schwerster Depressionsformen gelten, die sehr umstritten war und mittlerweile zum Standardangebot verschiedener Kliniken gehört, nicht zuletzt wegen der guten Compliance. In diesem Sinne sind Leitlinien eo ipso innovationsträge und dokumentieren stets den aktuell gesicherten Wissensstand, nicht aber neuere Ansätze.

\section{Implementierung von Leitlinien in einer jugend- psychiatrischen Klinik}

Trotz der Übung im multiaxialen Diagnostizieren und der teilweise erfolgten Manualisierung auch von psychodynamisch orientierten Therapieansätzen ist die klinische Arbeit nicht ohne eine explizite Einführung und Schulung möglich, ähnlich wie bei AMDP, OPD (-KJ) oder standardisierten Testverfahren.

Besonders in komplexen Fällen ist die Akzeptanz der klinischen Anwender in unserer Institution hoch, nicht zuletzt wegen der prinzipiell interdisziplinären Anwendbarkeit, die auch nicht-medizinische oder psychologische Mitarbeiter an der Entwicklung einer Diagnose und eines Therapieplans teilhaben lässt und damit die Transparenz erhöht. Ein nur scheinbarer Mehraufwand bei der Einführung wird schnell durch eine bessere Fundierung der Behandlung und geringere interdisziplinäre Kommunikationsprobleme gerade bei problematischen Fällen ausgeglichen.

\section{Implementierung in ein klinikinternes Qualitäts- managementsystem}

Neben der oben skizzierten primär fachlichen Integration von Leitlinien in klinische Arbeitskontexte erfüllen diese auch eine wichtige Funktion im Rahmen eines QM-Systems bzw. einzelner QM-Vorhaben. Das klinikinterne QM-Projekt «Depression und Suicidalität» (Leitung Dr. med. Joerg Burmeister), das sieben Standards zu diesem Themenkomplex erarbeitet hat, benutzt im Bereich Jugendpsychiatrie (Leitung Dr. med. Sibille Kühnel) die Leitlinien als Orientierung und Basis für auf die jeweiligen stationären Gegebenheiten fokussierte Handlungsanweisungen. Die störungsbezogenen Leitlinien werden in diesem Prozess in setting- und mitarbeiterorientierte Leitlinien transformiert und spezifiziert.

Somit gelingt eine an den aktuellen wissenschaftlichen und fachlichen Diskurs anknüpfende Integration von externer Evidenz in die internen Arbeitsprozesse. Durch die Gewährleistung einer nachhaltigen und regelhaften Überarbeitung im Sinne der kontinuierlichen Qualitätsverbesserung (TQM) beider Ansätze ist eine sichere Basis für alle Mitarbeiter, Patienten und Angehörigen erreicht und die stationsbezogenen Konzepte und Therapieverträge können in einem nächsten Schritt durch störungsbezogene Module ergänzt werden.

Im günstigen Fall ergibt sich ein wechselseitig befruchtender Austausch und die Integration klinischempirischen und wissenschaftlich-empirischen Wissens und Könnens.

\section{Ausblick und Zukunftsperspektiven}

Anhand der Leitlinien für episodische Depressionen der drei deutschen Fachgesellschaften für Kinderund Jugendpsychiatrie wurde ein Ansatz im Qualitätsmanagement skizziert, der es erlaubt, evidenzbasierte und störungsspezifische fachliche Leitlinien mit institutions- und settingbezogenen Standards zu verbinden.

Diese Vorgehensweise erscheint insofern interessant, als die in vielen QM-Modellen eher im Hintergrund stehende Verknüpfung von internen und externen QM-Ansätzen anhand konkreter und patientenbezogener Störungsszenarien erreicht werden kann und so ein wertvoller Beitrag zur Sicherung der Prozessqualität geleistet werden kann. Noch günstiger wären selbstverständlich eine aus den besonderen regionalen und soziokulturellen Hintergründen einer Versorgungslandschaft hervorgehende Leitlinienentwicklung und transnationale Vergleiche.

Denn die Erfahrung lehrt, dass die Akzeptanz und damit Relevanz aller Leitlinien dadurch entscheidend mitbestimmt wird, dass die späteren Anwender auch 
an verantwortlicher Stelle bei der Erarbeitung beteiligt waren. Hier sind für den Schweizer Raum insbesondere die profunden Erfahrungen im ambulanten und niedergelassenen Bereich noch nicht adäquat ausgeschöpft, womit sich abschliessend die vertraute Frage nach der Ressourcenverteilung innerhalb des Qualitätsmanagements auch für diese zentrale fachliche Aufgabe stellt.

\section{Literatur}

1 Swertz P, Möller J. EFQM-System auf Erfolgskurs. krankenhaus umschau 1998;68:396-9.

2 Sackett DL, Richardson WS, Rosenberg W, Haynes RB. Evidence-based medicine. Edinburgh: Churchill Livingstone; 1998.

3 Remschmidt H, Schmidt MH (Hrsg.). Das multiaxiale Klassifikationsschema für psychische Störungen im Kindesund Jugendalter nach ICD 10 der WHO. 3. Auflage. Bern: Huber; 1994

4 Döpfner M, Lehmkuhl G. Zur Notwendigkeit von Qualitätsstandards in der Kinder- und Jugendpsychiatrie. Z Kinderund Jugendpsych 1993;21:188-93.

5 Schmeck K, Poustka F, Katschnig H. Qualitätssicherung und Lebensqualität in der Kinder- und Jugendpsychiatrie. Wien: Springer; 1998.

6 Deutsche Gesellschaft für Kinder- und Jugendpsychiatrie und Psychotherapie, Bundesarbeitsgemeinschaft leitender Klinikärzte für Kinder- und Jugendpsychiatrie und Psychotherapie, Berufsverband der Ärzte für Kinder- und Jugendpsychiatrie und Psychotherapie (Hrsg.). Leitlinien zu Diagnostik und Therapie von psychischen Störungen im Säuglings-, Kindesund Jugendalter. Köln: Deutscher Ärzteverlag; 2000. 